\title{
Integral Equations for Memory Functions Involving Projection Operators*
}

\author{
A. Z. Akcasu \\ Department of Nuclear Engineering, The University of Michigan, Ann Arbor, Michigan 48105 \\ (Received 5 July 1972)

\begin{abstract}
Kinetic equations for the phase-space-time correlation functions contain memory functions that involve projection operators. It is shown that these memory functions can be represented by integral equations involving only real-time correlation functions, thereby eliminating the projection operators completely in the kinetic description of correlation functions. The weak-coupling and density expansions of the memory functions have been obtained through these integral equations.
\end{abstract}

\section{INTRODUCTION}

The calculation of the self- and density-correla tion functions in simple classical fluids via a kinetic equation, or a generalized Langevin equation, ${ }^{1,2}$ has received considerable attention in the past few years. ${ }^{3-10}$ It is shown in these studies that the self-correlation function ${ }^{3}$

$S_{s}\left(\overrightarrow{\mathrm{p}}, \overrightarrow{\mathrm{p}}^{\prime}, t\right) \equiv\left\langle g_{\alpha}(\overrightarrow{\mathrm{p}}, t) g_{\alpha}^{*}\left(\overrightarrow{\mathrm{p}}^{\prime}, 0\right)\right\rangle-\left\langle g_{\alpha}(\overrightarrow{\mathrm{p}}, t)\right\rangle\left\langle g_{\alpha}\left(\overrightarrow{\mathrm{p}}^{\prime}, 0\right)\right\rangle$,

where

$$
\begin{aligned}
& \oint_{\alpha}(\overrightarrow{\mathrm{p}}, t)=\delta\left(\overrightarrow{\mathrm{p}}-\overrightarrow{\mathrm{p}}^{\alpha}(t)\right) e^{i \overrightarrow{\mathrm{k}} \cdot \overrightarrow{\mathrm{x}}^{\alpha}(t)}, \\
& \left\langle g_{\alpha}(\overrightarrow{\mathrm{p}}, t)\right\rangle=\delta_{\mathrm{KR}}(\overrightarrow{\mathrm{k}}) M(\overrightarrow{\mathrm{p}}),
\end{aligned}
$$

and $\overrightarrow{\mathrm{x}}^{\alpha}(t)$ and $\overrightarrow{\mathrm{p}}^{\alpha}(t)$ are the position and momentum of the test particle $(\alpha)$, satisfies the following kinetic equation:

$$
\begin{aligned}
\left(\frac{\partial}{\partial t}\right. & \left.-\frac{i \overrightarrow{\mathrm{k}} \cdot \overrightarrow{\mathrm{p}}}{m}\right) \oint_{s}\left(\overrightarrow{\mathrm{p}}, \overrightarrow{\mathrm{p}}^{\prime}, t\right) \\
& =-\int_{0}^{t} d u \int d \overrightarrow{\mathrm{p}}^{\prime \prime} \varphi_{s}\left(\overrightarrow{\mathrm{p}}, \overrightarrow{\mathrm{p}}^{\prime \prime}, u\right) \mathrm{G}_{s}\left(\overrightarrow{\mathrm{p}}^{\prime \prime}, \overrightarrow{\mathrm{p}}^{\prime}, t-u\right) .
\end{aligned}
$$

In (1) and below $\langle[\cdots]\rangle$ denotes the thermal average of the quantity $[\cdots], \delta_{\mathrm{KR}}(\overrightarrow{\mathrm{k}})$ is the Kroenecker $\delta$ and $M(\overrightarrow{\mathrm{p}})$ is the Maxwellian distribution. The memory function $\varphi_{s}\left(\vec{p}, \vec{p}^{\prime}, t\right)$ in (2) is defined in Laplace domain by

$\varphi_{s}\left(\overrightarrow{\mathrm{p}}, \overrightarrow{\mathrm{p}}^{\prime}, s\right)=\frac{1}{M\left(\overrightarrow{\mathrm{p}}^{\prime}\right)}\left\langle\sigma_{\alpha}^{*}\left(\overrightarrow{\mathrm{p}}^{\prime}\right) \frac{1}{s-(1-P) i L} \sigma_{\alpha}(\overrightarrow{\mathrm{p}})\right\rangle$,

where

$$
\begin{aligned}
& \sigma_{\alpha}(\overrightarrow{\mathrm{p}})=\sum_{\beta \neq \alpha} \sigma_{\alpha \beta}(\overrightarrow{\mathrm{p}}), \\
& \sigma_{\alpha \beta}(\overrightarrow{\mathrm{p}})=\frac{\partial}{\partial \overrightarrow{\mathrm{p}}} \cdot \frac{\partial V\left(\left|\overrightarrow{\mathrm{x}}^{\alpha \beta}\right|\right)}{\partial \overrightarrow{\mathbf{x}}^{\alpha \beta}} \delta\left(\overrightarrow{\mathrm{p}}-\overrightarrow{\mathrm{p}}^{\alpha}\right) e^{i \overrightarrow{\mathrm{x}} \cdot \overrightarrow{\mathbf{x}}^{\alpha}}, \\
& \overrightarrow{\mathbf{x}}^{\alpha \beta}=\overrightarrow{\mathbf{x}}^{\alpha}-\overrightarrow{\mathrm{x}}^{\beta},
\end{aligned}
$$

and $V(x)$ is the interparticle potential. The projection operator $P$ in (3) is defined ${ }^{3}$ by its action on a phase function $G$ with zero mean:

$$
P G=\int d \overrightarrow{\mathrm{p}} \frac{\left\langle G g_{\alpha}^{*}(\overrightarrow{\mathrm{p}})\right\rangle}{M(\overrightarrow{\mathrm{p}})} g_{\alpha}(\overrightarrow{\mathrm{p}}) \text {. }
$$

It has also been shown that the phase-spacedensity correlation function ${ }^{4}$

$$
\begin{aligned}
g\left(\overrightarrow{\mathrm{p}}, \overrightarrow{\mathrm{p}}^{\prime}, t\right)=\left\langle g(\overrightarrow{\mathrm{p}}, t) g *\left(\overrightarrow{\mathrm{p}}^{\prime}, 0\right)\right\rangle \\
-\langle g(\overrightarrow{\mathrm{p}}, t)\rangle\left\langle g\left(\overrightarrow{\mathrm{p}}^{\prime}, 0\right)\right\rangle,
\end{aligned}
$$

where

and

$$
g(\overrightarrow{\mathrm{p}}, t)=\sum_{\alpha} g_{\alpha}(\overrightarrow{\mathrm{p}}, t)
$$

$$
\frac{1}{V}\langle g(\overrightarrow{\mathrm{p}}, t)\rangle=n M(\overrightarrow{\mathrm{p}}) \delta_{\mathrm{KR}}(\overrightarrow{\mathrm{k}})
$$

satisfies

$$
\begin{aligned}
\left(\frac{\partial}{\partial t}\right. & \left.-\frac{i \overrightarrow{\mathrm{k}} \cdot \overrightarrow{\mathrm{p}}}{m}\right) \mathcal{S}\left(\overrightarrow{\mathrm{p}}, \overrightarrow{\mathrm{p}}^{\prime}, t\right)-\mu(\overrightarrow{\mathrm{p}}) \int d \overrightarrow{\mathrm{p}} \mathcal{G}\left(\overrightarrow{\mathrm{p}}, \overrightarrow{\mathrm{p}}^{\prime}, t\right) \\
& =-\int_{0}^{t} d u \int d \overrightarrow{\mathrm{p}}^{\prime \prime} \varphi\left(\overrightarrow{\mathrm{p}}, \overrightarrow{\mathrm{p}}^{\prime \prime}, u\right) \mathcal{G}\left(\overrightarrow{\mathrm{p}}^{\prime \prime}, \overrightarrow{\mathrm{p}}^{\prime}, t-u\right),
\end{aligned}
$$

where

$$
\mu(\overrightarrow{\mathrm{p}})=-\frac{i \overrightarrow{\mathrm{k}} \cdot \overrightarrow{\mathrm{p}}}{m} n M(\overrightarrow{\mathrm{p}}) C(k) .
$$

In (8), $C(k)$ and $n$ are the direct correlation function and the number density, respectively. The memory function $\varphi\left(\vec{p}, \vec{p}^{\prime}, t\right)$ in (7) is given by

$\varphi\left(\overrightarrow{\mathrm{p}}, \overrightarrow{\mathrm{p}}^{\prime}, s\right)$

$$
=\frac{1}{N M\left(\overrightarrow{\mathrm{p}}^{\prime}\right)}\left\langle\sigma^{*}\left(\overrightarrow{\mathrm{p}}^{\prime}\right) \frac{1}{s-(1-P) i L}(1-P) \sigma(\overrightarrow{\mathrm{p}})\right\rangle,
$$

where $N=n V$ is the number of particles in the system and

$$
\sigma(\overrightarrow{\mathrm{p}})=\sum_{\alpha} \sigma_{\alpha}(\overrightarrow{\mathrm{p}}) \text {. }
$$

The projection operator $P$ in $(9 a)$ is defined by its action on a phase function $G$ with zero mean $\mathrm{as}^{4}$

$$
P G=\left(\int d \overrightarrow{\mathrm{p}} \frac{\left\langle G g^{*}(\overrightarrow{\mathrm{p}})\right\rangle}{n M(\overrightarrow{\mathrm{p}})} g(\overrightarrow{\mathrm{p}})-C(k)\left\langle G \rho^{*}\right\rangle \rho\right) \frac{1}{V},
$$

where $\rho$ is the configuration-space number density

$$
\rho=\int d \overrightarrow{\mathrm{p}} g(\overrightarrow{\mathrm{p}})=\sum_{\alpha} e^{i \overrightarrow{\mathbf{k}} \cdot \overrightarrow{\mathrm{x}}^{\alpha}} .
$$

It is observed that the central problem in the calculation of the self- and density-correlation functions with the kinetic equations is to find meaning- 
ful and tractable approximations to the memory functions $\varphi_{s}\left(\overrightarrow{\mathrm{p}}, \overrightarrow{\mathrm{p}}^{\prime}, t\right)$ and $\varphi\left(\overrightarrow{\mathrm{p}}, \overrightarrow{\mathrm{p}}^{\prime}, t\right)$. It is to be noted that the time evolution of these functions is deter mined by the reduced propagator $e^{i t(1-P) L}$ rather than the conventional Liouville propagator $e^{i t L}$. In Refs. 3 and 4 we calculated $\varphi_{s}$ and $\varphi$ in the lowest order in the coupling coefficient, and showed that the resulting kernels were non-Markovian generalizations of the conventional Fokker-Planck operator. Forster and Martin ${ }^{5}$ worked out the weakcoupling expansion of $\varphi$ using diagrammatic techniques. More recently, Mazenko ${ }^{6}$ obtained an expansion of $\varphi$ to the lowest order in the density and showed that the resulting kernel is a generalization of the linearized Boltzmann collision operator to arbitrary wavelengths and frequencies. Boley ${ }^{7}$ has presented a shorter derivation of Mazenko's kernel.

In addition to these systematic perturbation expansions, several phenomenological models for $\varphi_{s}$ and $\varphi$ have been proposed in the recent litera ture to calculate the self-correlation and densitycorrelation functions at liquid densities in the $k$ and $w$ regions encountered in the neutron-scattering experiments. ${ }^{8-11}$

The objectives of this paper are to show that the memory functions can be related to the force correlation functions by integral equations, thereby eliminating the need of working with the reduced resolvent $[s-i(1-P) L]^{-1}$ and to develop expansions for $\varphi_{s}$ and $\varphi$ in the density and coupling constant using these integral equations. The integral equations obtained in this paper are extensions of those derived by $\mathrm{Mori}^{2}$ in matrix form to the continuous index representation of the state variables.

\section{INTEGRAL EQUATION FOR THE SELF-CORRELATION MEMORY FUNCTION $\varphi_{s}\left(\overrightarrow{\mathrm{p}}, \overrightarrow{\mathrm{p}}^{\prime}, t\right)$}

We use the operator identity

$\frac{1}{s-(1-P) i L}=\frac{1}{s-i L}-\frac{1}{s-i L}$ PiL $\frac{1}{s-(1-P) i L}$

in (3) and express $\varphi_{s}\left(\overrightarrow{\mathrm{p}}, \overrightarrow{\mathrm{p}}^{\prime}, s\right)$ (we drop the subscript $s$ on $\varphi_{s}$ in this section) as

$$
\varphi\left(\overrightarrow{\mathrm{p}}, \overrightarrow{\mathrm{p}}^{\prime}, s\right)=\varphi_{0}\left(\overrightarrow{\mathrm{p}}, \overrightarrow{\mathrm{p}}^{\prime}, s\right)+\varphi_{1}\left(\overrightarrow{\mathrm{p}}, \overrightarrow{\mathrm{p}}^{\prime}, s\right),
$$

where

$$
\begin{aligned}
& \varphi_{0}\left(\overrightarrow{\mathrm{p}}, \overrightarrow{\mathrm{p}}^{\prime}, s\right)=\frac{1}{M\left(\overrightarrow{\mathrm{p}}^{\prime}\right)}\left\langle\sigma_{\alpha}^{*}\left(\overrightarrow{\mathrm{p}}^{\prime}\right) \frac{1}{s-i L} \sigma_{\alpha}(\overrightarrow{\mathrm{p}})\right\rangle, \\
& \varphi_{1}\left(\overrightarrow{\mathrm{p}}, \overrightarrow{\mathrm{p}}^{\prime}, s\right)=-\frac{1}{M\left(\overrightarrow{\mathrm{p}}^{\prime}\right)}\left\langle\sigma_{\alpha}^{*}\left(\overrightarrow{\mathrm{p}}^{\prime}\right) \frac{1}{s-i L} P i L \frac{1}{s-i L} \sigma_{\alpha}(\overrightarrow{\mathrm{p}})\right\rangle .
\end{aligned}
$$

Substituting the definition of $P$ from (5) into (14), one finds

$$
\begin{aligned}
& \varphi_{1}\left(\overrightarrow{\mathrm{p}}, \overrightarrow{\mathrm{p}}^{\prime}, s\right) \\
& \quad=\frac{1}{M\left(\overrightarrow{\mathrm{p}}^{\prime}\right)} \int d \overrightarrow{\mathrm{p}}^{\prime \prime}\left(\left\langle g_{\alpha}^{*}\left(\overrightarrow{\mathrm{p}}^{\prime \prime}\right) i L \frac{1}{s-i(1-P) L} \sigma_{\alpha}(\overrightarrow{\mathrm{p}})\right\rangle\right.
\end{aligned}
$$

$$
\left.\times \frac{1}{M\left(\overrightarrow{\mathrm{p}}^{\prime \prime}\right)}\left\langle\sigma_{\alpha}^{*}\left(\overrightarrow{\mathrm{p}}^{\prime}\right) \frac{1}{s-i L} g_{\alpha}\left(\overrightarrow{\mathrm{p}}^{\prime \prime}\right)\right\rangle\right) .
$$

Using the self-adjointness of $L$ in the first factor of the integrand, $i L g_{\alpha}\left(\overrightarrow{\mathrm{p}}^{\prime \prime}\right)=\sigma_{\alpha}\left(\overrightarrow{\mathrm{p}}^{\prime \prime}\right)+\left(i \overrightarrow{\mathrm{k}} \cdot \overrightarrow{\mathrm{p}}^{\prime \prime} / m\right)$ $\times g_{\alpha}\left(\overrightarrow{\mathrm{p}}^{\prime \prime}\right)$, and $\left\langle g^{*}\left(\overrightarrow{\mathrm{p}}^{\prime \prime}\right)[s-i(1-P) L]^{-1} \sigma_{\alpha}(\overrightarrow{\mathrm{p}})\right\rangle=0$, we can verify that

$$
\frac{1}{M\left(\overrightarrow{\mathrm{p}}^{\prime \prime}\right)}\left\langle g_{\alpha}^{*}\left(\overrightarrow{\mathrm{p}}^{\prime \prime}\right) i L \frac{1}{s-i(1-P) L} \sigma_{\alpha}(\overrightarrow{\mathrm{p}})\right\rangle=-\varphi\left(\overrightarrow{\mathrm{p}}, \overrightarrow{\mathrm{p}}^{\prime \prime}, s\right) \text {. }
$$

This is a crucial identity in the derivation of the integral equation for $\varphi\left(\overrightarrow{\mathrm{p}}, \overrightarrow{\mathrm{p}}^{\prime}, s\right)$.

The second factor in the integrand of (15) can be related to $\varphi_{0}\left(\overrightarrow{\mathrm{p}}, \overrightarrow{\mathrm{p}}^{\prime}, s\right)$ in (13) by observing that

$$
\begin{aligned}
\left\langle\sigma_{\alpha}^{*}\left(\overrightarrow{\mathrm{p}}^{\prime}\right)\right. & \left.\frac{1}{s-i L} \sigma_{\alpha}(\overrightarrow{\mathrm{p}})\right\rangle \\
& =\left\langle\sigma_{\alpha}^{*}\left(\overrightarrow{\mathrm{p}}^{\prime}\right) \frac{1}{s-i L}\left(i L g_{\alpha}(\overrightarrow{\mathrm{p}})-\frac{i \overrightarrow{\mathrm{k}} \cdot \overrightarrow{\mathrm{p}}}{m} g_{\alpha}(\overrightarrow{\mathrm{p}})\right)\right\rangle \\
& =\left(s-\frac{i \overrightarrow{\mathrm{k}} \cdot \overrightarrow{\mathrm{p}}}{m}\right)\left\langle\sigma_{\alpha}^{*}\left(\overrightarrow{\mathrm{p}}^{\prime}\right) \frac{1}{s-i L} g_{\alpha}(\overrightarrow{\mathrm{p}})\right\rangle,
\end{aligned}
$$

where we have used $\left\langle\sigma_{\alpha}^{*}\left(\overrightarrow{\mathrm{p}}^{\prime}\right) g_{\alpha}(\overrightarrow{\mathrm{p}})\right\rangle=0$, which can be verified by calculating the indicated ensemble average explicitly. Substituting (16) and (17) into (15), we obtain $\varphi_{1}\left(\overrightarrow{\mathrm{p}}, \overrightarrow{\mathrm{p}}^{\prime}, s\right)$ in terms of $\varphi\left(\overrightarrow{\mathrm{p}}, \overrightarrow{\mathrm{p}}^{\prime}, s\right)$ and $\varphi_{0}\left(\overrightarrow{\mathrm{p}}, \overrightarrow{\mathrm{p}}^{\prime}, s\right)$. Using this result in (12), we arrive at the desired integral equation for $\varphi_{s}\left(\overrightarrow{\mathrm{p}}, \overrightarrow{\mathrm{p}}^{\prime}, s\right)$ :

$$
\begin{aligned}
\varphi_{s}\left(\overrightarrow{\mathrm{p}}, \overrightarrow{\mathrm{p}}^{\prime}, s\right)= & \varphi_{0}\left(\overrightarrow{\mathrm{p}}, \overrightarrow{\mathrm{p}}^{\prime}, s\right)+\int d p^{\prime \prime} \varphi_{s}\left(\overrightarrow{\mathrm{p}}, \overrightarrow{\mathrm{p}}^{\prime \prime}, s\right) \\
& \times \varphi_{0}\left(\overrightarrow{\mathrm{p}}^{\prime \prime}, \overrightarrow{\mathrm{p}}^{\prime}, s\right)\left(s-\frac{i \overrightarrow{\mathrm{k}} \cdot \overrightarrow{\mathrm{p}}^{\prime \prime}}{m}\right)^{-1} .
\end{aligned}
$$

[See Appendix for an alternative derivation and discussion of (18).]

This equation relates $\varphi_{s}\left(\overrightarrow{\mathrm{p}}, \overrightarrow{\mathrm{p}}^{\prime}, t\right)$ to $\varphi_{0}\left(\overrightarrow{\mathrm{p}}, \overrightarrow{\mathrm{p}}^{\prime}, t\right)$, which evolves in time according to the Liouville propagator, as indicated by (13). Expressing the latter as

$$
\varphi_{0}\left(\overrightarrow{\mathrm{p}}, \overrightarrow{\mathrm{p}}^{\prime}, t\right)=\frac{1}{M\left(\overrightarrow{\mathrm{p}}^{\prime}\right)}\left\langle\sigma_{\alpha}(\overrightarrow{\mathrm{p}}, t) \sigma_{\alpha}^{*}\left(\overrightarrow{\mathrm{p}}^{\prime}, 0\right)\right\rangle,
$$

we find that $\varphi_{0}$ is proportional to the time-correlation function of $\sigma_{\alpha}(\overrightarrow{\mathrm{p}}, t)$ and $\sigma_{\alpha}(\overrightarrow{\mathrm{p}}, 0)$. We can use (18) to obtain expansions of $\varphi_{s}\left(\overrightarrow{\mathrm{p}}, \overrightarrow{\mathrm{p}}^{\prime}, t\right)$ in a small parameter $\epsilon$ (not necessarily a power-series expansion) in terms of the expansion coefficients of $\varphi_{0}\left(\overrightarrow{\mathrm{p}}, \overrightarrow{\mathrm{p}}^{\prime}, t\right)$ in the same parameter. Let us suppose that $\varphi_{0}$ has an expansion as ${ }^{12}$

$$
\varphi_{0}=\epsilon^{n}\left(a_{0}+\epsilon a_{1}+\epsilon^{2} a_{2}+\cdots\right),
$$

where $a_{f}$ are of course functions of $\overrightarrow{\mathrm{p}}, \overrightarrow{\mathrm{p}}^{\prime}$, and $t$. Substituting this into (18) we find

$$
\begin{aligned}
\varphi_{s}\left(\overrightarrow{\mathrm{p}}, \overrightarrow{\mathrm{p}}^{\prime}, s\right)= & \epsilon^{n}\left(a_{0}+\epsilon a_{1}+\cdots+a_{n-1} \epsilon^{n-1}\right) \\
& +\epsilon^{2 n}\left[a_{n}+\int d \overrightarrow{\mathrm{p}}^{\prime \prime} a_{0}\left(\overrightarrow{\mathrm{p}}, \overrightarrow{\mathrm{p}}^{\prime \prime}, s\right) a_{0}\left(\overrightarrow{\mathrm{p}}^{\prime \prime}, \overrightarrow{\mathrm{p}}^{\prime}, s\right)\right.
\end{aligned}
$$




$$
\left.\times\left(s-\frac{i \overrightarrow{\mathrm{k}} \cdot \overrightarrow{\mathrm{p}}}{m}\right)^{-1}\right]+O\left(\epsilon^{2 n+1}\right) .
$$

One conclusion from this result is that $\varphi_{0}$ and $\varphi_{s}$ have the same expansion coefficients up to $\epsilon^{2 n-1}$. The effect of the integral term in (18) which arises from the projection operator $P$ in the expression of $\varphi_{s}\left(\vec{p}, \vec{p}^{\prime}, s\right)$ [cf. Eq. (3)] appears first in the term of the order of $\epsilon^{2 n}$. As an application, we consider the weak-coupling and low-density expansions. The weak-coupling expansion of $\varphi_{0}\left(\overrightarrow{\mathrm{p}}, \overrightarrow{\mathrm{p}}^{\prime}, s\right)$ immediately follows from (13) by noting that $\sigma_{\alpha}(\vec{p})$ is of the or$\operatorname{der} \lambda$, the interaction strength, and expanding $\left(s-i L_{0}-i L^{\prime}\right)^{-1}$ in powers of $\lambda$ :

$$
\varphi_{0}\left(\overrightarrow{\mathrm{p}}, \overrightarrow{\mathrm{p}}^{\prime}, s\right)=\lambda^{2}\left(a_{0}+\lambda a_{1}+\lambda^{2} a_{2}+\cdots\right),
$$

where

$a_{0}\left(\overrightarrow{\mathrm{p}}, \overrightarrow{\mathrm{p}}^{\prime}, s\right)=\frac{1}{M\left(\overrightarrow{\mathrm{p}}^{\prime}\right)}\left\langle\sigma_{\alpha}^{*}\left(\overrightarrow{\mathrm{p}}^{\prime}\right) \frac{1}{s-i L_{0}} \sigma_{\alpha}(\overrightarrow{\mathrm{p}})\right\rangle$,

$a_{1}\left(\overrightarrow{\mathrm{p}}, \overrightarrow{\mathrm{p}}^{\prime}, s\right)$

$$
=\frac{1}{M\left(\overrightarrow{\mathrm{p}}^{\prime}\right)}\left\langle\sigma_{\alpha}^{*}\left(\overrightarrow{\mathrm{p}}^{\prime}\right) \frac{1}{s-i L_{0}} i L^{\prime} \frac{1}{s-i L_{0}} \sigma_{\alpha}(\overrightarrow{\mathrm{p}})\right\rangle, \ldots .
$$

Hence, the weak-coupling expansion of $\varphi_{s}\left(\vec{p}, \vec{p}^{\prime}, s\right)$ is

$$
\begin{aligned}
\varphi_{s}\left(\overrightarrow{\mathrm{p}}, \overrightarrow{\mathrm{p}}^{\prime}, s\right) & =\lambda^{2}\left(a_{0}+\lambda a_{1}\right) \\
+\lambda^{4} & {\left[a_{2}+\int d \overrightarrow{\mathrm{p}}^{\prime \prime} a_{0}\left(\overrightarrow{\mathrm{p}}, \overrightarrow{\mathrm{p}}^{\prime \prime}, s\right) a_{0}\left(\overrightarrow{\mathrm{p}}^{\prime \prime}, \overrightarrow{\mathrm{p}}^{\prime}, s\right)\right.} \\
& \left.\times\left(s-\frac{i \overrightarrow{\mathrm{k}} \cdot \overrightarrow{\mathrm{p}}^{\prime \prime}}{m}\right)^{-1}\right]+O\left(\lambda^{5}\right)
\end{aligned}
$$

In Ref. 3, $a_{0}\left(\overrightarrow{\mathrm{p}}, \overrightarrow{\mathrm{p}}^{\prime}, t\right)$ was evaluated explicitly [cf. Eqs. (60)-(64) of Ref. 3] using (21b). The calculation of $a_{1}$ and $a_{2}$ does not seem to be of any interest at present, and thus will not be attempted.

The density expansion of $\varphi_{0}\left(\vec{p}, \vec{p}^{\prime}, s\right)$ can be achieved using the following form ${ }^{13,14}$ of the cluster expansion of $G(s) \equiv(s-i L)^{-1}$ :

$$
G(s)=G_{\alpha \gamma}(s)+\sum_{j \neq \alpha, \gamma}\left[G_{\alpha \gamma j}(s)-G_{\alpha \gamma}(s)\right]+\cdots,
$$

where

$$
G_{\alpha \gamma}(s)=\left(s-i L_{\alpha \gamma}-i L_{(N-2)}^{0}\right)^{-1},
$$

$$
G_{\alpha \gamma j}(s)=\left(s-i L_{\alpha \gamma j}-i L_{(N-3)}^{0}\right)^{-1} .
$$

In (23b) $L_{\alpha \gamma}$ is the Liouville operator involving the particles of $\alpha$ and $\gamma$ and $i L_{(N-2)}^{0}$ is the streaming operator involving the remaining $(N-2)$ particles. Similarly $L_{\alpha \gamma f}$ in (23c) is the Liouville operator involving the particles $\alpha, \gamma$, and $j$ and $i L_{(N-3)}^{0}$ is the streaming operator associated with the remaining $(N-3)$ particles. Substituting (23a) into (13), and using (4a), we obtain

where

$$
\varphi_{0}\left(\overrightarrow{\mathrm{p}}, \overrightarrow{\mathrm{p}}^{\prime}, s\right)=n\left(b_{0}+n b_{1}+\cdots\right),
$$

$$
\begin{aligned}
& b_{0}\left(\overrightarrow{\mathrm{p}}, \overrightarrow{\mathrm{p}}^{\prime}, s\right)= \frac{V}{M\left(\overrightarrow{\mathrm{p}}^{\prime}\right)}\left\langle\sigma_{12}^{*}\left(\overrightarrow{\mathrm{p}}^{\prime}\right) \frac{1}{s-i L_{12}} \sigma_{12}(\overrightarrow{\mathrm{p}})\right\rangle, \\
& b_{1}\left(\overrightarrow{\mathrm{p}}, \overrightarrow{\mathrm{p}}^{\prime}, s\right)=\frac{V^{2}}{M\left(\overrightarrow{\mathrm{p}}^{\prime}\right)}\left(\left\langle\left[\sigma_{12}^{*}\left(\overrightarrow{\mathrm{p}}^{\prime}\right)+\sigma_{13}^{*}\left(\overrightarrow{\mathrm{p}}^{\prime}\right)\right] \frac{1}{s-i L_{123}} \sigma_{12}(\overrightarrow{\mathrm{p}})\right\rangle\right. \\
&\left.\quad-\left\langle\sigma_{12}^{*}\left(\overrightarrow{\mathrm{p}}^{\prime}\right) \frac{1}{s-i L_{12}} \sigma_{12}(\overrightarrow{\mathrm{p}})\right\rangle\right) .
\end{aligned}
$$

It follows from (19) to (20) with the help of (24) that $\varphi_{s}\left(\overrightarrow{\mathrm{p}}, \overrightarrow{\mathrm{p}}^{\prime}, s\right)$ has the following density expansion:

$$
\begin{aligned}
\varphi_{s}\left(\overrightarrow{\mathrm{p}}, \overrightarrow{\mathrm{p}}^{\prime}, s\right)= & n b_{0}+n^{2}\left[b_{1}+\int d \overrightarrow{\mathrm{p}}^{\prime \prime} b_{0}\left(\overrightarrow{\mathrm{p}}, \overrightarrow{\mathrm{p}}^{\prime \prime}, s\right)\right. \\
& \left.\times b_{0}\left(\overrightarrow{\mathrm{p}}^{\prime \prime}, \overrightarrow{\mathrm{p}}^{\prime}, s\right)\left(s-\frac{i \overrightarrow{\mathrm{k}} \cdot \overrightarrow{\mathrm{p}}^{\prime \prime}}{m}\right)^{-1}\right]+O\left(n^{3}\right) .
\end{aligned}
$$

The matrix element in (25) can be evaluated explicitly by expressing $L_{12}\left(\overrightarrow{\mathrm{p}}^{1}, \overrightarrow{\mathrm{x}}^{1}, \overrightarrow{\mathrm{p}}^{2}, \overrightarrow{\mathrm{x}}^{2}\right)$ in terms of the center-of-mass coordinate $\vec{R}$ and the relative coordinate $\vec{r}=\vec{x}^{1}-\vec{x}^{2}$ :

$$
\begin{aligned}
& i L_{12}=\frac{\overrightarrow{\mathrm{p}}}{m} \cdot \frac{\partial}{\partial \overrightarrow{\mathrm{R}}}+i L(\overrightarrow{\mathrm{q}}, \overrightarrow{\mathrm{r}}), \\
& i L(\overrightarrow{\mathrm{q}}, \overrightarrow{\mathrm{r}})=\frac{\overrightarrow{\mathrm{q}}}{\frac{1}{2} m} \cdot \frac{\partial}{\partial \overrightarrow{\mathrm{r}}}-\frac{\partial V(r)}{\partial \overrightarrow{\mathrm{r}}} \cdot \frac{\partial}{\partial \overrightarrow{\mathrm{q}}},
\end{aligned}
$$

where

$$
\overrightarrow{\mathrm{P}}=\frac{1}{2}\left(\overrightarrow{\mathrm{p}}^{1}+\overrightarrow{\mathrm{p}}^{2}\right), \quad \overrightarrow{\mathrm{q}}=\frac{1}{2}\left(\overrightarrow{\mathrm{p}}^{1}-\overrightarrow{\mathrm{p}}^{2}\right), \quad \overrightarrow{\mathrm{R}}=\frac{1}{2}\left(\overrightarrow{\mathrm{x}}^{1}+\overrightarrow{\mathrm{x}}^{2}\right) .
$$

Substituting $\sigma_{12}(\overrightarrow{\mathrm{p}})$ from (4a) into (25) and performing the indicated thermal averages, we find the expression of $\varphi_{s}$ in the lowest order in density as

$$
\begin{aligned}
\varphi_{s}\left(\overrightarrow{\mathrm{p}}, \overrightarrow{\mathrm{p}}^{\prime}, s\right)= & \frac{n}{M\left(\overrightarrow{\mathrm{p}}^{\prime}\right)}\left(\frac{\beta}{\pi m}\right)^{3} \frac{\partial}{\partial \overrightarrow{\mathrm{p}}} \frac{\partial}{\partial \overrightarrow{\mathrm{p}}^{\prime}}: \int d \vec{\alpha} \int d \overrightarrow{\mathrm{r}} \int d \overrightarrow{\mathrm{q}} e^{-\beta\left(\alpha^{2}+\alpha^{2}\right) / m} \\
& \times\left[g(r) \frac{d V(r)}{d r} e^{-i \overrightarrow{\mathrm{k}} \cdot \overrightarrow{\mathrm{r}} / 2} \delta\left(\overrightarrow{\mathrm{p}}^{\prime}-\vec{\alpha}-\overrightarrow{\mathrm{q}}\right)\left(s-\frac{i \overrightarrow{\mathrm{k}} \cdot \vec{\alpha}}{m}-i L(\overrightarrow{\mathrm{q}}, \overrightarrow{\mathrm{r}})\right)^{-1} \frac{d V(r)}{d r} e^{i \mathbf{k} \cdot \mathrm{r} / 2} \delta(\overrightarrow{\mathrm{p}}-\vec{\alpha}-\overrightarrow{\mathrm{q}})\right]+O\left(n^{2}\right)
\end{aligned}
$$

We have not attempted at present to calculate the second-order terms in the expansion of $\varphi_{s}$. This would require the evaluation of the matrix element $b_{1}\left(\overrightarrow{\mathrm{p}}, \overrightarrow{\mathrm{p}}^{\prime}, s\right)$ given by $(26)$ which involves triple collisions.

\section{INTEGRAL EQUATION FOR THE DENSITY-} CORRELATION MEMORY FUNCTION $\varphi\left(\overrightarrow{\mathbf{p}}, \overrightarrow{\mathbf{p}}^{\prime}, s\right)$

The derivation of the integral equation for the memory function $\varphi\left(\overrightarrow{\mathrm{p}}, \overrightarrow{\mathrm{p}}^{\prime}, t\right)$ appearing the kinetic equation (7) for the density correlation function 
proceeds in the same way as discussed in Sec. II. We again split $\varphi$, defined in (9a), as $\varphi=\varphi_{0}+\varphi_{1}$, using (11), where

$\varphi_{0}\left(\overrightarrow{\mathrm{p}}, \overrightarrow{\mathrm{p}}^{\prime}, s\right)=\frac{1}{N M\left(\overrightarrow{\mathrm{p}}^{\prime}\right)}\left\langle\sigma^{*}\left(\overrightarrow{\mathrm{p}}^{\prime}\right) \frac{1}{s-i L}(1-P) \sigma(\overrightarrow{\mathrm{p}})\right\rangle$

and

$\varphi_{1}\left(\overrightarrow{\mathrm{p}}, \overrightarrow{\mathrm{p}}^{\prime}, s\right)=\frac{-1}{N M\left(\overrightarrow{\mathrm{p}}^{\prime}\right)}$

$$
\times\left\langle\sigma^{*}\left(\overrightarrow{\mathrm{p}}^{\prime}\right) \frac{1}{s-i L} P i L \frac{1}{s-(1-P) i L}(1-P) \sigma(\overrightarrow{\mathrm{p}})\right\rangle .
$$

Using the definition of the projection operator $P$ given by (10a) in (31), we find

$$
\varphi_{1}\left(\overrightarrow{\mathrm{p}}, \overrightarrow{\mathrm{p}}^{\prime}, s\right)=\int d \overrightarrow{\mathrm{p}}^{\prime \prime} \varphi\left(\overrightarrow{\mathrm{p}}, \overrightarrow{\mathrm{p}}^{\prime \prime}, s\right) q_{0}\left(\overrightarrow{\mathrm{p}}^{\prime \prime}, \overrightarrow{\mathrm{p}}^{\prime}, s\right),
$$

where

$$
q_{0}\left(\overrightarrow{\mathrm{p}}, \overrightarrow{\mathrm{p}}^{\prime}, s\right)=\frac{1}{N M\left(\overrightarrow{\mathrm{p}}^{\prime}\right)}\left\langle\sigma^{*}\left(\overrightarrow{\mathrm{p}}^{\prime}\right) \frac{1}{s-i L} g(\overrightarrow{\mathrm{p}})\right\rangle .
$$

We then express $\varphi_{0}$ in (30) in terms of $q_{0}$ by first replacing $(1-P) \sigma(\overrightarrow{\mathrm{p}})$ by $(1-P) i \operatorname{Lg}(\overrightarrow{\mathrm{p}})$, and using the following step:

$$
\begin{aligned}
\varphi_{\mathrm{C}}=\frac{1}{N M\left(\overrightarrow{\mathrm{p}}^{\prime}\right)}\left(\left\langle\sigma^{*}\left(\overrightarrow{\mathrm{p}}^{\prime}\right)\right.\right. & \left.\left(\frac{s}{s-i L}-1\right) g(\overrightarrow{\mathrm{p}})\right\rangle \\
& \left.-\left\langle\sigma^{*}\left(\overrightarrow{\mathrm{p}}^{\prime}\right) \frac{1}{s-i L} \operatorname{PiLg}(\overrightarrow{\mathrm{p}})\right\rangle\right)
\end{aligned}
$$

Evaluating PiL $g(\overrightarrow{\mathrm{p}})$ using (10a) and noting

$$
\begin{aligned}
q_{0}\left(\overrightarrow{\mathrm{p}}, \overrightarrow{\mathrm{p}}^{\prime}, t=0\right) & =\frac{1}{N M\left(\overrightarrow{\mathrm{p}}^{\prime}\right)}\left\langle\sigma^{*}\left(\overrightarrow{\mathrm{p}}^{\prime}\right) g(\overrightarrow{\mathrm{p}})\right\rangle \\
& =\frac{i \overrightarrow{\mathrm{k}} \cdot \overrightarrow{\mathrm{p}}}{m} M(\overrightarrow{\mathrm{p}}) n g(k),
\end{aligned}
$$

we find

$$
\begin{array}{r}
\varphi_{0}\left(\overrightarrow{\mathrm{p}}, \overrightarrow{\mathrm{p}}^{\prime}, s\right)=\left(s-\frac{i \overrightarrow{\mathrm{k}} \cdot \overrightarrow{\mathrm{p}}}{m}\right) q_{0}\left(\overrightarrow{\mathrm{p}}, \overrightarrow{\mathrm{p}}^{\prime}, s\right)-q_{0}\left(\overrightarrow{\mathrm{p}}, \overrightarrow{\mathrm{p}}^{\prime}, t=0\right) \\
-\mu(\overrightarrow{\mathrm{p}}) \int d \overrightarrow{\mathrm{p}} q_{0}\left(\overrightarrow{\mathrm{p}}, \overrightarrow{\mathrm{p}}^{\prime}, s\right), \quad \text { (35a) }
\end{array}
$$

which in time domain reads

$$
\begin{aligned}
\varphi_{0}\left(\overrightarrow{\mathrm{p}}, \overrightarrow{\mathrm{p}}^{\prime}, t\right)=\left(\frac{\partial}{\partial t}-\frac{i \overrightarrow{\mathrm{k}} \cdot \overrightarrow{\mathrm{p}}}{m}\right) q_{0}\left(\overrightarrow{\mathrm{p}}, \overrightarrow{\mathrm{p}}^{\prime}, t\right) \\
-\mu(\overrightarrow{\mathrm{p}}) \int d \overrightarrow{\mathrm{p}} q_{0}\left(\overrightarrow{\mathrm{p}}, \overrightarrow{\mathrm{p}}^{\prime}, t\right) .
\end{aligned}
$$

The desired integral equation is found by substituting (32) into $\varphi=\varphi_{0}+\varphi_{1}$ as

$$
\begin{aligned}
\varphi\left(\overrightarrow{\mathrm{p}}, \overrightarrow{\mathrm{p}}^{\prime}, s\right)= & \varphi_{0}\left(\overrightarrow{\mathrm{p}}, \overrightarrow{\mathrm{p}}^{\prime}, s\right) \\
& +\int d \overrightarrow{\mathrm{p}}^{\prime \prime} \varphi\left(\overrightarrow{\mathrm{p}}, \overrightarrow{\mathrm{p}}^{\prime \prime}, s\right) q_{0}\left(\overrightarrow{\mathrm{p}}^{\prime \prime}, \overrightarrow{\mathrm{p}}^{\prime}, s\right) .
\end{aligned}
$$

Although it is possible to express $q_{0}$ in terms of $\varphi_{0}$ by solving (35), we find it more convenient to work with both $q_{0}$ and $\varphi_{0}$.

The weak-coupling expansion of $\varphi$ is obtained from (36) by first finding the expansions of $\varphi_{0}$ and $q_{0}$ in the coupling constant $\lambda$ as

$$
\begin{aligned}
& q_{0}=\lambda\left(b_{0}+\lambda b_{1}+\cdots\right), \\
& \varphi_{0}=\lambda^{2}\left(a_{0}+\lambda a_{1}+\cdots\right) .
\end{aligned}
$$

The expansion coefficients $b_{0}, b_{1}, \ldots$ are obtained from (33) by expanding $\left(s-i L_{0}-i L^{\prime}\right)^{-1}$ :

$$
\begin{aligned}
& b_{0}\left(\overrightarrow{\mathrm{p}}, \overrightarrow{\mathrm{p}}^{\prime}, s\right) \equiv \frac{1}{N M\left(\overrightarrow{\mathrm{p}}^{\prime}\right)}\left\langle\sigma^{*}\left(\overrightarrow{\mathrm{p}}^{\prime}\right) \frac{1}{s-i L_{0}} g(\overrightarrow{\mathrm{p}})\right\rangle \\
& \quad=\left(s-\frac{i \overrightarrow{\mathrm{k}} \cdot \overrightarrow{\mathrm{p}}}{m}\right)^{-1} \frac{1}{N M\left(\overrightarrow{\mathrm{p}}^{\prime}\right)}\left\langle\sigma^{*}\left(\overrightarrow{\mathrm{p}}^{\prime}\right) \frac{1}{s-i L_{0}} \sigma(\overrightarrow{\mathrm{p}})\right\rangle, \\
& b_{1}\left(\overrightarrow{\mathrm{p}}, \overrightarrow{\mathrm{p}}^{\prime}, s\right) \equiv \frac{1}{N M\left(\overrightarrow{\mathrm{p}}^{\prime}\right)}\left\langle\sigma^{*}\left(\overrightarrow{\mathrm{p}}^{\prime}\right) \frac{1}{s-i L_{0}} i L^{\prime} \frac{1}{s-i L_{0}} g(\overrightarrow{\mathrm{p}})\right\rangle \\
& \quad=\left(s-\frac{i \overrightarrow{\mathrm{k}} \cdot \overrightarrow{\mathrm{p}}}{m}\right)^{-1} \frac{1}{N M\left(\overrightarrow{\mathrm{p}}^{\prime}\right)}\left\langle\sigma^{*}\left(\overrightarrow{\mathrm{p}}^{\prime}\right) \frac{1}{s-i L_{0}} \sigma(\overrightarrow{\mathrm{p}})\right\rangle .
\end{aligned}
$$

The coefficients $a_{0}, a_{1}, \ldots$ can be obtained either directly from their definition in (30) using $P \sigma(\vec{p})$ $=\mu(\vec{p}) \rho$, or by substituting (37a) into the integral equation (35) and noting that $\mu(\overrightarrow{\mathrm{p}})$ is at least of the order of $\lambda$. We present only the lowest-order term in the coupling explicitly as

$$
\begin{aligned}
a_{0}\left(\overrightarrow{\mathrm{p}}, \overrightarrow{\mathrm{p}}^{\prime}, s\right)= & \frac{1}{N M\left(\overrightarrow{\mathrm{p}}^{\prime}\right)}\left\langle\sigma^{*}\left(\overrightarrow{\mathrm{p}}^{\prime}\right) \frac{1}{s-i L_{0}}(1-P) \sigma(\overrightarrow{\mathrm{p}})\right\rangle \\
= & \frac{1}{N M\left(\overrightarrow{\mathrm{p}}^{\prime}\right)}\left(\left\langle\sigma^{*}\left(\overrightarrow{\mathrm{p}}^{\prime}\right) \frac{1}{s-i L_{0}} \sigma(\overrightarrow{\mathrm{p}})\right\rangle\right. \\
& \left.-\mu(\overrightarrow{\mathrm{p}})\left\langle\sigma^{*}\left(\overrightarrow{\mathrm{p}}^{\prime}\right) \frac{1}{s-i L_{0}} \rho\right\rangle\right) .
\end{aligned}
$$

The relation between $a_{0}, a_{1}, \ldots$ and $b_{0}, b_{1}, \ldots$ is determined by (38) as

$$
\begin{aligned}
& a_{0}=\left(s-i \frac{\overrightarrow{\mathrm{k}} \cdot \overrightarrow{\mathrm{p}}}{m}\right) b_{1}-\mu(\overrightarrow{\mathrm{p}}) \int d \overrightarrow{\mathrm{p}} b_{0}\left(\overrightarrow{\mathrm{p}}, \overrightarrow{\mathrm{p}}^{\prime}, s\right), \\
& a_{1}=\left(s-\frac{i \overrightarrow{\mathrm{k}} \cdot \overrightarrow{\mathrm{p}}}{m}\right) b_{2}-\mu(\overrightarrow{\mathrm{p}}) \int d \overrightarrow{\mathrm{p}} b_{1}\left(\overrightarrow{\mathrm{p}}, \overrightarrow{\mathrm{p}}^{\prime}, s\right) .
\end{aligned}
$$

Substituting (37) into (36) we find the desired weakcoupling expansion of $\varphi$ as

$$
\begin{aligned}
\varphi\left(\overrightarrow{\mathrm{p}}, \overrightarrow{\mathrm{p}}^{\prime}, s\right)=\lambda^{2}\left\{a_{0}+\lambda\right. & {\left[a_{1}+\int d \overrightarrow{\mathrm{p}}^{\prime \prime} a_{0}\left(\overrightarrow{\mathrm{p}}, \overrightarrow{\mathrm{p}}^{\prime \prime}, s\right)\right.} \\
\times & \left.\left.b_{0}\left(\overrightarrow{\mathrm{p}}^{\prime \prime}, \overrightarrow{\mathrm{p}}^{\prime}, s\right)\right]\right\}+O\left(\lambda^{4}\right) .
\end{aligned}
$$

The lowest-order term $a_{0}\left(\overrightarrow{\mathrm{p}}, \overrightarrow{\mathrm{p}}^{\prime}, t\right)$ was calculated explicitly as a function of time in a previous work. ${ }^{4}$ In Eq. (42) we display the next higher term without attempting to evaluate it explicitly.

The density expansion of $\varphi\left(\vec{p}, \vec{p}^{\prime}, s\right)$ follows the exact same pattern. We first expand $q_{0}$ and $\varphi_{0}$ using the cluster expansion of $G(s)$ in (23):

$$
q_{0}=n\left(c_{0}+n c_{1}+n^{2} c_{2}+\cdots\right),
$$




$$
\varphi_{0}=n\left(d_{0}+n d_{1}+n^{2} d_{2}+\cdots\right),
$$

where

$$
\begin{aligned}
c_{0}=\frac{V}{M\left(\overrightarrow{\mathrm{p}}^{\prime}\right)} & \left\langle\sigma_{12}^{*}\left(\overrightarrow{\mathrm{p}}^{\prime}\right) \frac{1}{s-i L_{12}} \sum_{j=1}^{2} g_{j}(\overrightarrow{\mathrm{p}})\right\rangle, \\
c_{1}=\frac{V^{2}}{M\left(\overrightarrow{\mathrm{p}}^{\prime}\right)} & \left(\left\langle\sigma_{12}^{*}\left(\overrightarrow{\mathrm{p}}^{\prime}\right) \frac{1}{s-i L_{123}} \sum_{j=1}^{3} g_{j}(\overrightarrow{\mathrm{p}})\right\rangle\right. \\
& \left.-\left\langle\sigma_{12}^{*}\left(\overrightarrow{\mathrm{p}}^{\prime}\right) \frac{1}{s-i L_{12}} \sum_{j=1}^{2} g_{j}(\overrightarrow{\mathrm{p}})\right\rangle\right),
\end{aligned}
$$

and

$$
d_{0}=\frac{V}{M(\overrightarrow{\mathrm{p}})}\left\langle\sigma_{12}^{*}\left(\overrightarrow{\mathrm{p}}^{\prime}\right) \frac{1}{s-i L_{12}}\left[\sigma_{12}(\overrightarrow{\mathrm{p}})+\sigma_{21}(\overrightarrow{\mathrm{p}})\right]\right\rangle .
$$

The expression of $d_{1}$ is lengthy, and will not be presented explicitly. However we can express $d_{1}$ as well as $d_{0}$ in terms of $c_{0}$ and $c_{1}$ again using (38):

$$
\begin{aligned}
& d_{0}=\left(s-\frac{i \overrightarrow{\mathrm{k}} \cdot \overrightarrow{\mathrm{p}}}{m}\right) c_{0}\left(\overrightarrow{\mathrm{p}}, \overrightarrow{\mathrm{p}}^{\prime}, s\right)-c_{0}\left(\overrightarrow{\mathrm{p}}, \overrightarrow{\mathrm{p}}^{\prime}, t=0\right), \\
& d_{1}=\left(s-\frac{i \overrightarrow{\mathrm{k}} \cdot \overrightarrow{\mathrm{p}}}{m}\right) c_{1}-\frac{\mu(\overrightarrow{\mathrm{p}})}{n} \int d \overrightarrow{\mathrm{p}} c_{0}\left(\overrightarrow{\mathrm{p}}, \overrightarrow{\mathrm{p}}^{\prime}, s\right) .
\end{aligned}
$$

The density expansion of $\varphi$ is now found by substituting (43) into (36):

$$
\begin{aligned}
\varphi\left(\overrightarrow{\mathrm{p}}, \overrightarrow{\mathrm{p}}^{\prime}, s\right)=n\left\{d_{0}+n[\right. & d_{1}+\int d \overrightarrow{\mathrm{p}}^{\prime \prime} d_{0}\left(\overrightarrow{\mathrm{p}}, \overrightarrow{\mathrm{p}}^{\prime \prime}, s\right) \\
& \left.\left.\times c_{0}\left(\overrightarrow{\mathrm{p}}^{\prime \prime}, \overrightarrow{\mathrm{p}}^{\prime}, s\right)\right]+\cdots\right\} .
\end{aligned}
$$

It is interesting to note the resemblance of the memory function $\varphi_{s}\left(\overrightarrow{\mathrm{p}}, \overrightarrow{\mathrm{p}}^{\prime}, s\right)$ and $\varphi\left(\overrightarrow{\mathrm{p}}, \overrightarrow{\mathrm{p}}^{\prime}, s\right)$ in the lowest order in density, which are given by (25) and (46), respectively. The latter involves $\sigma_{12}(\overrightarrow{\mathrm{p}})$ $+\sigma_{21}(\vec{p})$, whereas $\varphi_{s}$ involves only $\sigma_{12}(\vec{p})$ in the indicated correlation functions. The lowest-order approximation to $\varphi\left(\vec{p}, \vec{p}^{\prime}, s\right)$ in density can be calculated as in (29):

$$
\begin{aligned}
& \varphi\left(\overrightarrow{\mathrm{p}}, \overrightarrow{\mathrm{p}}^{\prime}, s\right)=\frac{n}{M\left(\overrightarrow{\mathrm{p}}^{\prime}\right)}\left(\frac{\beta}{m \pi}\right)^{3} \frac{\partial}{\partial \overrightarrow{\mathrm{p}}} \frac{\partial}{\partial \overrightarrow{\mathrm{p}}^{\prime}}: \int d \vec{\alpha} \int d \overrightarrow{\mathrm{r}} \int d \overrightarrow{\mathrm{q}} e^{-\beta\left(\alpha^{2}+q^{2}\right) / m} \\
& \times\left[g(r) \frac{d V(r)}{d r}\left[e^{-i \overrightarrow{\mathrm{k}} \cdot \overrightarrow{\mathrm{r}} / 2} \delta\left(\overrightarrow{\mathrm{p}}^{\prime}-\vec{\alpha}-\overrightarrow{\mathrm{q}}\right)-e^{i \overrightarrow{\mathbf{k}} \cdot \overrightarrow{\mathbf{r}} / 2} \delta\left(\overrightarrow{\mathrm{p}}^{\prime}-\vec{\alpha}+\overrightarrow{\mathrm{q}}\right)\right]\right. \\
& \left.\times\left(s-\frac{i \overrightarrow{\mathrm{k}} \cdot \vec{\alpha}}{m}-i L(\overrightarrow{\mathrm{q}}, \overrightarrow{\mathrm{r}})\right)^{-1} \frac{d V(r)}{d r} e^{i \overrightarrow{\mathrm{k}} \cdot \overrightarrow{\mathrm{r}} / 2} \delta(\overrightarrow{\mathrm{p}}-\vec{\alpha}-\overrightarrow{\mathrm{q}})\right]+O\left(n^{2}\right)
\end{aligned}
$$

This expression is identical to that obtained by Mazenko ${ }^{6}$ and later by Boley. ${ }^{7}$

\section{CONCLUSIONS}

The main conclusion of this paper is that the memory functions involving reduced propagators $e^{i t(1-P) L}$ can be expressed in terms of the realtime correlation functions through certain integral equations. Although the original kinetic equations are obtained using the projection-operator technique, the projection operator has been eliminated from the kinetic description of the time-correlation functions by the integral equation representation of the memory functions. Since the reduced resolvent $[s-i(1-P) L]^{-1}$ does not lend itself easily to conventional perturbative analysis, the integral equations obtained in this paper provide a more convenient starting point for the perturbation approximation to the memory functions; this we demonstrated in the paper by finding the first two coefficients in the weak-coupling and density expansions.

The work presented in this paper is regarded as an extension and application of the integral equation derived by Mori [Eq. (3.14) and (6.38) of Ref. $2]$ in discrete-matrix notation. As in the derivation of the kinetic equation itself, ${ }^{4}$ we have ex- tended Mori's formalism to a continuous index representation of the dynamical variables to obtain the integral equations for the memory functions, the continuous index being the momentum variable. The idea of expressing the memory functions in terms of mechanical correlation functions was also pointed out by Martin. ${ }^{15}$

\section{APPENDIX: AN ALTERNATIVE DERIVATION AND DISCUSSION OF THE INTEGRAL EQUATION (18)}

The correlation function $\varphi_{0}\left(\overrightarrow{\mathrm{p}}, \overrightarrow{\mathrm{p}}^{\prime}, s\right)$ defined by (13) can be related to the self-correlation function $\oint_{s}\left(\vec{p}, \vec{p}^{\prime}, s\right)$ using a similar procedure given in (17):

$$
\begin{aligned}
\varphi_{0}\left(\overrightarrow{\mathrm{p}}, \overrightarrow{\mathrm{p}}^{\prime}, s\right)= & \left(s-\frac{i \overrightarrow{\mathrm{k}} \cdot \overrightarrow{\mathrm{p}}}{m}\right)\left[\delta\left(\overrightarrow{\mathrm{p}}-\overrightarrow{\mathrm{p}}^{\prime}\right)\right. \\
& \left.-\frac{1}{M\left(\overrightarrow{\mathrm{p}}^{\prime}\right)}\left(s-\frac{i \overrightarrow{\mathrm{k}} \cdot \overrightarrow{\mathrm{p}}^{\prime}}{m}\right) \Theta_{s}\left(\overrightarrow{\mathrm{p}}, \overrightarrow{\mathrm{p}}^{\prime}, s\right)\right] .
\end{aligned}
$$

In order to compress the writing below, we introduce the following functions:

$$
\begin{aligned}
& Z_{0}\left(\overrightarrow{\mathrm{p}}, \overrightarrow{\mathrm{p}}^{\prime}, s\right) \equiv\left(s-\frac{i \overrightarrow{\mathrm{k}} \cdot \overrightarrow{\mathrm{p}}}{m}\right)^{-1} \varphi_{0}\left(\overrightarrow{\mathrm{p}}, \overrightarrow{\mathrm{p}}^{\prime}, s\right), \\
& \mathcal{H}_{s}\left(\overrightarrow{\mathrm{p}}, \overrightarrow{\mathrm{p}}^{\prime}, s\right) \equiv \frac{1}{M\left(\overrightarrow{\mathrm{p}}^{\prime}\right)}\left(s-\frac{i \overrightarrow{\mathrm{k}} \cdot \overrightarrow{\mathrm{p}}^{\prime}}{m}\right) \mathcal{S}_{s}\left(\overrightarrow{\mathrm{p}}, \overrightarrow{\mathrm{p}}^{\prime}, s\right) .
\end{aligned}
$$


In terms of these new functions (A1) reads as

$$
Z_{0}\left(\overrightarrow{\mathrm{p}}, \overrightarrow{\mathrm{p}}^{\prime}, s\right)=\delta\left(\overrightarrow{\mathrm{p}}-\overrightarrow{\mathrm{p}}^{\prime}\right)-\mathcal{H}_{s}\left(\overrightarrow{\mathrm{p}}, \overrightarrow{\mathrm{p}}^{\prime}, s\right) \text {. }
$$

The generalized Langevin equation (2) for $g_{s}\left(\vec{p}, \vec{p}^{\prime}\right.$, $s$ ) on the other hand can be cast in Laplace domain into

$$
\begin{aligned}
\mathcal{F}_{s}\left(\overrightarrow{\mathrm{p}}, \overrightarrow{\mathrm{p}}^{\prime}, s\right)= & \delta\left(\overrightarrow{\mathrm{p}}-\overrightarrow{\mathrm{p}}^{\prime}\right) \\
& -\int d^{3} p^{\prime \prime} Z_{s}\left(\overrightarrow{\mathrm{p}}, \overrightarrow{\mathrm{p}}^{\prime \prime}, s\right) \mathcal{H}_{s}\left(\overrightarrow{\mathrm{p}}^{\prime \prime}, \overrightarrow{\mathrm{p}}^{\prime}, s\right),
\end{aligned}
$$

where

$$
Z_{s}\left(\overrightarrow{\mathrm{p}}, \overrightarrow{\mathrm{p}}^{\prime}, s\right) \equiv\left(s-\frac{i \overrightarrow{\mathrm{k}} \cdot \overrightarrow{\mathrm{p}}}{m}\right)^{-1} \varphi_{s}\left(\overrightarrow{\mathrm{p}}, \overrightarrow{\mathrm{p}}^{\prime}, s\right)
$$

If we eliminate $\mathfrak{H}_{s}\left(\overrightarrow{\mathrm{p}}, \overrightarrow{\mathrm{p}}^{\prime}, s\right)$ in favor of $Z_{0}\left(\overrightarrow{\mathrm{p}}, \overrightarrow{\mathrm{p}}^{\prime}, s\right)$ between (A4) and (A5), we obtain

$$
\begin{aligned}
Z_{s}\left(\overrightarrow{\mathrm{p}}, \overrightarrow{\mathrm{p}}^{\prime}, s\right)= & Z_{0}\left(\overrightarrow{\mathrm{p}}, \overrightarrow{\mathrm{p}}^{\prime}, s\right) \\
& +\int d^{3} p^{\prime \prime} Z_{s}\left(\overrightarrow{\mathrm{p}}, \overrightarrow{\mathrm{p}}^{\prime \prime}, s\right) Z_{0}\left(\overrightarrow{\mathrm{p}}^{\prime \prime}, \overrightarrow{\mathrm{p}}^{\prime}, s\right),
\end{aligned}
$$

which is identical to (18). This equation can be regarded as an integral equation for $Z_{0}\left(\overrightarrow{\mathrm{p}}, \overrightarrow{\mathrm{p}}^{\prime}, s\right)$ if we assume that the memory function $Z_{s}\left(\overrightarrow{\mathrm{p}}, \overrightarrow{\mathrm{p}}^{\prime}, s\right)$ is known. In this sense it is formally equivalent to the original generalized Langevin equation (A5) for the self-correlation function $\mathcal{F}_{s}\left(\overrightarrow{\mathrm{p}}, \overrightarrow{\mathrm{p}}^{\prime}, s\right)$. Since $Z_{0}\left(\overrightarrow{\mathrm{p}}, \overrightarrow{\mathrm{p}}^{\prime}, s\right)$ and $\mathcal{H}_{s}\left(\overrightarrow{\mathrm{p}}, \overrightarrow{\mathrm{p}}^{\prime}, s\right)$ are simply related to each other by (A4), we can investigate in principle the self-correlation function by either of these two integral equations provided $Z_{s}\left(\overrightarrow{\mathrm{p}}, \overrightarrow{\mathrm{p}}^{\prime}, s\right)$ is known. However, we can also regard (A7) as an integral equation for $Z_{s}\left(\overrightarrow{\mathrm{p}}, \overrightarrow{\mathrm{p}}^{\prime}, s\right)$, as we have implied in the text, provided that we can find a meaningful approximation to $Z_{0}\left(\overrightarrow{\mathrm{p}}, \overrightarrow{\mathrm{p}}^{\prime}, s\right)$, such as the low-density and weak-coupling approximations. By virtue of the simple relation between $Z_{0}\left(\overrightarrow{\mathrm{p}}, \overrightarrow{\mathrm{p}}^{\prime}, s\right)$ and $\mathfrak{H}_{s}(\overrightarrow{\mathrm{p}}$, $\left.\overrightarrow{\mathrm{p}}^{\prime}, s\right)$, this procedure is formally equivalent to approximating $\mathcal{H}_{s}\left(\overrightarrow{\mathrm{p}}, \overrightarrow{\mathrm{p}}^{\prime}, s\right)$ in the original kinetic equation to find a corresponding approximation to the memory $Z_{s}\left(\mathrm{p}, \mathrm{p}^{\prime}, s\right)$. The method used by Boley $^{6}$ to obtain the Mazenko's kernel ${ }^{7}$ is essential ly the same as this procedure.
*Work supported in part by a grant from the National Science Foundation, under Contract No. GK-19360X.

${ }^{1} \mathrm{R}$. Zwanzig, in Lectures in Theoretical Physics, edited by W. E. Brittin (Wiley, New York, 1961).

${ }^{2}$ H. Mori, Progr. Theoret. Phys. (Kyoto) 33, 423 (1965)

${ }^{3}$ A. Z. Akcasu, N. Corngold, and J. J. Duderstadt, Phys. Fluids, 13, 2213 (1970).

${ }^{4}$ A. Z. Akcasu and J. J. Duderstadt, Phys. Rev. 188 , 479 (1969).

${ }^{5}$ D. Forster and P. C. Martin, Phys. Rev. A 2,1575 (1970).

${ }^{6}$ G. F. Mazenko, Phys. Rev. A $\underline{3}, 2121$ (1971).

${ }^{7}$ C. D. Boley, Phys. Rev. A $\underline{5}, \overline{9} 86$ (1972).

${ }^{8}$ P. Ortoleva and P. C. Martin, Phys. Rev. A $\underline{2}, 1575$ (1970).

${ }^{9}$ R. C. Desai, Phys. Rev. A $\underline{3}, 329$ (1971).

${ }^{10}$ J. J. Duderstadt and A. Z. Akcasu, Phys. Rev. A 1 , 905 (1970); 2, 1097 (1970).

${ }^{11}$ A. Z. Akcasu and E. J. Linnebur, Proceedings of the
IAEA Fifth Symposium on Neutron Inelastic Scattering, Grenoble, France, March, 1972 (unpublished).

${ }^{12}$ Since the purpose of the subsequent analysis is mainly to show how certain results obtained by other methods (Refs. 6 and 7) readily follow from the projection operator formalism, we shall not attempt to discuss the question of divergences associated with such expansions. However, we think that the memory function $\varphi_{s}$ determined by (18) can still have an expansion as in (20), even $\varphi_{0}$ cannot be expanded as in (19), as a result of the additional terms arising from the projection operator in the evolution of $\varphi_{s}$.

${ }^{13}$ M. H. Ernst, in Lectures in Theoretical Physics, Kinetic Theory, edited by W. E. Brittin (Gordon and Breach, New York, 1967), Vol. IXC, p. 426.

${ }^{14}$ This expansion can be obtained very elegantly using the partition algebra discussed by $\mathrm{C}$. Arf, K. Imre, and E. Ozizmir [J. Math. Phys., 6, 1179 (1965)].

${ }^{15}$ See the footnote on p. 447 of Ref. 2. 